\title{
Planning Premises and Design Considerations for Hybrid Operating Room
}

\author{
${ }^{1} \mathrm{~V}$ Siddharth, ${ }^{2}$ Sunil Kant, ${ }^{3} \mathrm{R}$ Chandrashekhar, ${ }^{4}$ Shakti Kumar Gupta
}

\begin{abstract}
The hybrid operating room can be defined as the combination of imaging system and operating table installed in an operating theater room for, e.g. use of an angiography imaging system and operation table in an operation theatre or use of an operating table in angiography room. Shorter patient recovery time, decreased length of stay, streamlined care delivery, improvement in cross-specialty communication, minimized risk for communication-related errors across clinical specialties and lower overall cost of care are some of the advantages of the hybrid operating room. Although no/limited data exists in the literature, the potential disadvantages of hybrid operating suite are cost, infection, prolonged anesthesia and radiation exposure. The primary components of a hybrid operating suite are an imaging system and imaging compatible operation table. Area required for hybrid operating suite varies from 80 to $150 \mathrm{~m}^{2}$. The most common configuration of hybrid operating suite includes a flat panel angiographic $\mathrm{X}$-ray imaging system and surgical equipment for cardiac surgery.
\end{abstract}

Keywords: Operating room, Hybrid operation room, Intraoperative imaging.

How to cite this article: Siddharth V, Kant S, Chandrashekhar R, Gupta SK. Planning Premises and Design Considerations for Hybrid Operating Room. Int J Res Foundation Hosp Healthc Adm 2014;2(1):50-56.

\section{Source of support: Nil \\ Conflict of interest: None}

\section{INTRODUCTION}

In recent years, we have seen 'winds of change', in medical science and nowhere can this be more aptly applied than

\footnotetext{
${ }^{1}$ Senior Resident, ${ }^{2}$ DDGAFMS (Coord)

${ }^{3}$ Chief Architect, ${ }^{4} \mathrm{Head}$

${ }^{1}$ Department of Hospital Administration, All India Institute of Medical Sciences, New Delhi, India

${ }^{2}$ Ministry of Defence, Government of India, New Delhi, India

${ }^{3}$ Ministry of Health and Family Welfare, Government of India New Delhi, India

${ }^{4}$ Department of Hospital Administration and Medical Superintendent, Dr RP Centre for Ophthalmic Sciences, All India Institute of Medical Sciences, New Delhi, India
}

Corresponding Author: V Siddharth, Senior Resident Department of Hospital Administration, All India Institute of Medical Sciences, Ansari Nagar, New Delhi-110029, India Phone: 9013844255, e-mail: dr.siddharthmamc@gmail.com in the great changes that have developed in regard to the construction, and organization, of the modern operating theater suite. ' 'OT has been defined as that specialized facility of the hospital where lifesaving or life improving procedures are carried out on the human body by invasive methods under strict aseptic conditions in a controlled environment by specially trained personnel to promote healing and cure with maximum safety, comfort and economy'. ${ }^{2}$

Imaging has a long history in the operating room (OR). In the 1960s, X-ray units were mounted on the ceiling, as they might be today. But the surgeon had to go to an adjacent room to view the image, and images could only be stored for 10 minutes. Mobile $\mathrm{C}$-arms, introduced in the late 1960s, have been a mainstay of OR imaging. But more hospitals are finding these $\mathrm{C}$-arms are no longer meeting their OR imaging needs. Increasingly complex surgical and interventional approaches require more advanced imaging. In response, many larger facilities have replaced the conventional OR configuration with new configurations known as hybrid ORs. The word 'Hybrid' originally refers to the result of interbreeding between plants or animals of two different species. These combine surgical equipment and instrumentation for open procedures with a fixed and dedicated imaging system as well as an imaging-compatible surgical table, lights, and surgical booms to accommodate open, minimally invasive and interventional procedures. ${ }^{3}$ It can function as either a conventional operating theater, or as a radiology facility, but crucially allows intra- and postoperative on-table imaging and intervention, and overcomes many of the limitations of standard facilities.

\section{Need for Hybrid OR}

The introduction and rapid growth of minimally invasive surgery (MIS) has stimulated interest in OR design. Hospitals and surgi-centers have been inundated with requests for construction and renovations to facilitate the practice of MIS. Other surgical disciplines and endoscopic environments also recognized the potential to create a procedural environment that would better meet their specific needs. ${ }^{4}$

Trends are driving the need for more imaging in the OR because of following reasons: 
- A growing aging population means more people are living longer to require surgery best performed with image guidance.

- More than $60 \%$ of all patients having surgical procedures are overweight or obese and often have comorbid diabetes and cardiovascular disease.

These trends increase the likelihood of more complications during interventional procedures, often leading to the need to convert to an open surgical procedure ${ }^{3}$ and moreover:

- The lines between interventional and surgical specialties are blurring - interventional procedures are becoming more complex, and surgical procedures are becoming less invasive.

- Increasing numbers of patients with complex disease are forcing cardiac surgeons and interventional cardiologists to collaborate more frequently.

More complex procedures in the interventional suite mean patients will require more clinical supervision and possibly a more intense level of care. This has been an important factor in shift from conventional operation theaters toward integrated and hybrid operating theaters. Following table describes the advantages and disadvantages of hybrid operating room (Table 1).

\section{Procedures Which can be performed in Hybrid Operating Theater}

Because of its versatile nature it can be used by various clinical specialities mainly for endovascular surgery, interventional radiology, open vascular surgery, open surgical and radiological procedures (Graph 1). ${ }^{5}$

\section{Planning and Design Challenges}

Following are the challenges associated with designing of hybrid operating room:

1. Multiple stakeholders

2. Required space

3. Co-ordination with multiple vendors

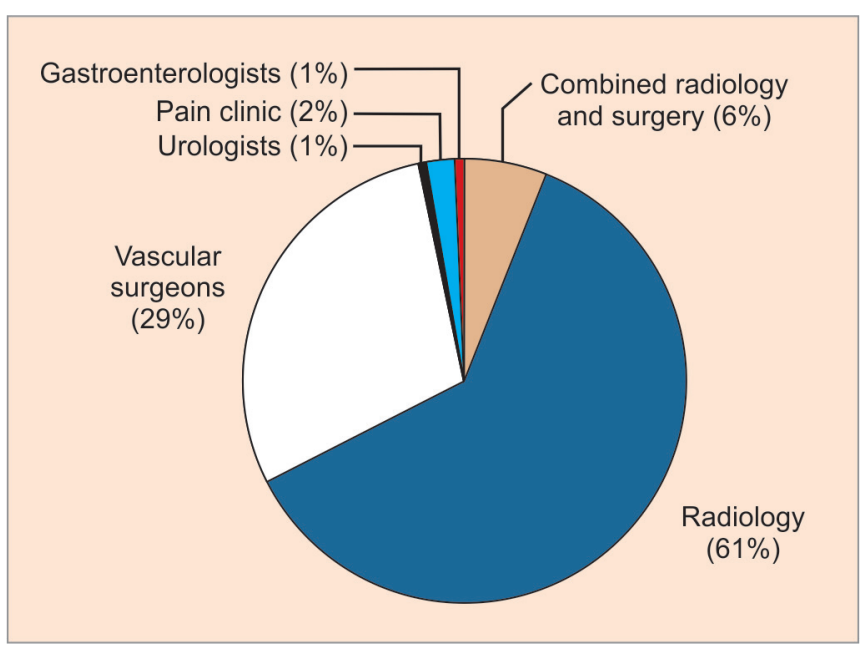

Graph 1: To demonstrate multispecialty usage of the hybrid theater ${ }^{5}$

a. Lighting

b. Monitors and video switching

c. X-ray/imaging system

d. Other OR requirement

4. Radiation safety

5. Capital cost.

\section{Planning for Hybrid OR}

From the outset, it should be recognized that effective design of a hybrid operating suite is a complex process which in most cases will require at least 6 months to over a year. The operating theater suite must be so well designed and so attractively set out, that not only will the work done there be of the highest order, but the personnel engaged therein, will find it a real pleasure to work in such ideal surroundings. Fatigue will be minimal, boredom nonexistent, efficiency at its highest peak, and pride of achievement the driving force. ${ }^{1}$

Before planning a hybrid operating room, a clear vision for the utilization should be established. Planning of the hybrid room is truly an interdisciplinary task. It involves much more co-ordination and multiple departmental involvements than planning for a conventional OR or

Table 1: Advantages and disadvantages of hybrid $O R^{3,6}$

\begin{tabular}{l} 
Advantages \\
\hline Shorter patient recovery time \\
Decreased length of stay \\
Streamlined delivery of care \\
Overall lower cost of care \\
Potential for revenue growth \\
Minimized risk for communication-related errors across clinical \\
specialties \\
Effectiveness and efficiency in training, teaching and research \\
High quality imaging vastly superior to portable systems \\
Promotes the multidisciplinary process and allows for efficient \\
use of staff and equipment
\end{tabular}
Disadvantages

Cost

Infection risk

Prolonged general anesthetic

Prolonged radiation exposure 


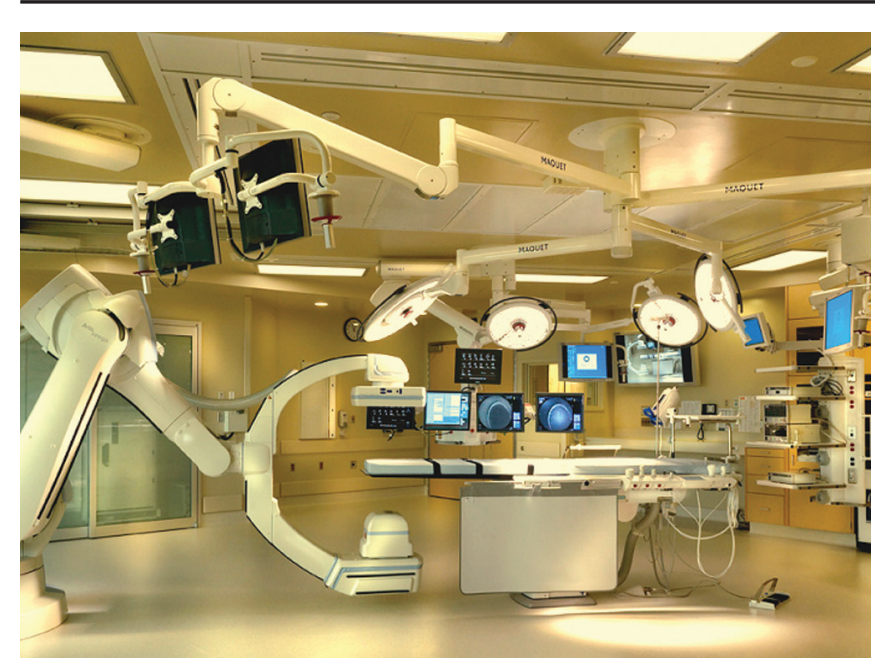

Fig. 1: Hybrid operating room (Providence Sacred Heart Medical Centre) ${ }^{11}$

catheterization laboratory alone. These rooms are used not only by surgeons and interventional cardiologists, but also by electro physiologists, neurosurgeons, and cardio thoracic vascular surgeons. ${ }^{7}$

Visits to established hybrid operating rooms, and discussions with experienced users help tremendously during the planning process. Key stakeholders need to be identified and for planning should be included at an early stage.

In most cases, the design team will be led by a vascular surgeon. In many cases, cardiac thoracic vascular surgery will also be interested and involved in the process of development of this suite. The multidisciplinary planning should have representation from hospital administration, anesthesiology, cardiology, neuroradiology, radiology, surgery, nursing staff, architects, engineering and radiology technologists who will be running the imaging equipment. ${ }^{8}$ Any specialty that would be using the OR needs to be involved in the planning. It is good to include the OR and catheterization laboratory equipment vendors chosen for the project so they can coordinate.

As each of these stake holders will be involved in the administration or actual procedures performed in the room, initial input is essential to prevent subsequent design flaws and friction. The construction of a hybrid suite requires a deep understanding of the technology and its implication for the surgical workflow.

\section{Hybrid Operating Suite - Design Considerations}

The primary components of the hybrid operating suite is imaging system as well as carefully designed operating tables to accommodate and optimize the usefulness of the radiographic equipment (Fig. 1). ${ }^{9}$

As such, conversion of established ORs to hybrid operating suites is usually not possible. A combination of two rooms or an OR with an adjacent storage space may be possible but clearly in the absence of new construction, creation of such a space in an established operating room can present considerable challenges. ${ }^{8}$ The expense of these constructions and modifications can vary up to $\$ 100,000$ depending on the original condition of the room, local contracting costs, architect's fees and companies that market fixed imaging systems. ${ }^{9}$

\section{Location}

The following things should be considered while deciding on the location of hybrid OR:10

- Relative ease of access to/from emergency department, radiology department, intensive care unit (ICU), inpatient beds, etc.

- Proximity to blood bank, labs, pharmacy, etc.

- Materials Support

- Supply restock support

- Standardize inventory

- Mobile or stationary storage.

- Flow management

- Ease of patient entry/exit

- Away from heavy traffic areas for sterility reasons.

- Adequate electrical service to support new imaging equipment.

\section{Size}

The size of the hybrid room should be able to accommodate: ${ }^{11}$

- Larger teams with additional diagnostic, imaging and surgical experts

- Additional equipment around 'typical' surgery equipment

- Provide image equipment control (sometimes with dedicated technicians) and storage areas outside of the OR itself

- Provide dedicated equipment support areas, some with their own temperature and air demands

- Allow mobility of equipment, as cost and use often require equipment to be shared between rooms

- Provide flexibility in equipment location and allow for unspecified future possibilities.

Usually a hybrid operating theater (OT) can be divided into various areas which includes procedure room of 80 $\mathrm{m}^{2}$, equipment room of $10 \mathrm{~m}^{2}$ and control room of $12 \mathrm{~m}^{2} .^{10}$ The OR should ideally be between 60 and $150 \mathrm{~m}^{2}$ with a minimum clear area of 40 to $50 \mathrm{~m}^{2} .{ }^{12}$ Another study says that OR should be between 55 and $90 \mathrm{~m}^{2}$, with a minimum clear area of 40 to $50 \mathrm{~m}^{2} .{ }^{9}$ Floor to ceiling height should be at minimum 10 feet to accommodate floor- or ceiling-mounted $\mathrm{C}$-arms capable of rotational angiography. ${ }^{9,12}$ Space for stor- 
age for the special procedure related equipment should be approximately 20 to $50 \mathrm{~m}^{2} .10$

\section{Radiation Protection}

The facility should be designed considering applicable laws, rules and regulations pertaining to radiation protection as the equipment utilized in hybrid operating suite can emit radiation. The walls of the hybrid OR requires lead lining to limit radiation exposure. ${ }^{11}$ Radiation exposure can be divided into exposure of the patient and exposure of personnel. It varies greatly between different procedures and is dependent on multiple factors. ${ }^{13}$

Most standard ORs have leaded covering $0.5 \mathrm{~mm}$ which is not sufficient for the radiation dose generated by a fixed unit. Lead-lined walls in the range of 2 to $3 \mathrm{~mm}$ for the fixed units may be needed. ${ }^{9,12}$ In addition, ceiling-mounted transparent shields are available for optimal scatter protection of the operator's upper body and face. Moreover, the newer portable $\mathrm{C}$-arm units are less restricted as to the energy they can deliver, compared to fixed systems. This implies an increasing imaging quality, but the boost setting on these units delivers radiation doses with intense scattered radiation. $^{14}$

\section{Operation Table}

Electronic integration of the table with the imaging system is essential to prevent collisions and to allow maximal capability of the imaging system. ${ }^{8}$ The preferred surgical operating table to accommodate such techniques should be preferably thin but highly stable and should provide complete clearance beneath a panning X-ray system. ${ }^{15}$ While many hospitals have adopted the use of mobile imaging tables with various levels of functionality (such as floating table tops), these tables are not satisfactory for modern imaging systems. ${ }^{8,16}$ The choice can be made between two fully integrated table systems. First, an operating table with a floating table top. Second, a conventional OR table system with two different, exchangeable table tops - a radiolucent one-piece carbon table top and a breakable table top.

Advantages of a carbon fiber table include reduction of radiation, improvement of the imaging quality and reduction of the radiation burden to personnel. When choosing for fixed carbon fiber table that can be controlled by a table-side console, one should realize that these tables are broad and therefore working in the groin area and at the lower extremities may be awkward. Patient transfers are less convenient using these broad tables. Modern tables, however, have a table top that can be moved longitudinally, away from the broad part, over such a distance that the patient easily is accessible. This requires sufficient space in the operation room, and anticipation when placing anesthetic equipment. Furthermore, one should realize that these 'radiologic' tables are not supplied with side bars on the narrow part; these are useful for connection of retractor systems or armrests. ${ }^{14}$

Finally, although an operation table that could bend in the middle would be useful, especially for hybrid procedures, such constructions can only be built by using metal nonradiopaque articulations, which are not available commercially. As a result, until now only two planes of tilt, craniocaudal and lateral have been possible. ${ }^{14}$

\section{Flat Screens and Monitors}

The hybrid suite must be equipped for continuous patient monitoring. A total of four to six ceiling-mounted flat screens as imaging tools for the procedures are necessary. Extreme care should be taken to ensure that these ceiling-mounted flat screens do not collide with operating lights. Monitors for the vital signs of the patient with provision for systemic arterial monitoring, central venous monitoring and continuous electrocardiographic surveillance is imperative. A large 40 to 56 inch flat panel should be available as well as cameras (wall/or in-light). ${ }^{12}$

\section{Audiovisual Systems}

Increased imaging requirements require an integrated audiovisual channeling system to allow various images to be moved to be different monitors throughout the room. For example, the ability to bring PACS system images (stores X-ray images) up to the operating table for review at the time of surgery is a useful adjunct as is the ability to bring the patient's physiologic information up to the surgeon's monitor if necessary. A variety of commercial systems are available to facilitate image routing throughout the operating room suite. ${ }^{8}$

\section{Imaging Technology}

The most common configuration includes a flat-panel angiographic X-ray imaging system and surgical equipment for open cardiac surgery. The second most common configuration is for interventional and surgical neuroapplications, although only a few hospitals have installed dedicated neuro-hybrid ORs. ${ }^{3}$ Imaging technology can include:

- Single-plane or biplane flat panel angiographic X-ray imaging system

- 3D rotational angiography

- Computed tomography scanning

- Intravascular ultrasound

- Duplex ultrasound

- 3D transesophageal echocardiogram 
- Magnetic resonance imaging

- Integrated robotic surgery

- Large, up to 83 inch image intensifiers.

\section{Equipment's in Hybrid Operating Room}

Equipment that may be installed in a hybrid OR (list not inclusive): ${ }^{3}$

- Surgical lights

- Surgical table

- Surgical (endoscopy) video systems

- Surgical booms

- Anesthesia system

- Robotic surgery system

- Magnetic catheter navigation system

- Heart-lung bypass system

- Intravascular and/or cardiac ultrasound system

- Operating microscope

- Neurosurgical navigation system.

\section{Image Acquisition and Display ${ }^{12}$}

In $\mathrm{CT}$ rotational angiography, which the latest hybrid imaging systems have, the $\mathrm{C}$-arm is used to rapidly rotate, obtaining serial images of the area in question in a radial fashion. The 3D reconstruction can be registered with subsequent real-time fluoroscopic images and projected to offer the clinician the ability to work in three dimensions. Data can be rendered volumetrically and overlayed on the fluoroscopic image, making the anatomy much more identifiable, a fused 2D/3D dataset can be created, or the information can be placed side by side. Further requirements of the suite's imaging system are a processing unit, a workstation, and a central image storage unit. The potential of any $\mathrm{C}$-arm equals the weakest link of each of these last three elements. While performing a procedure, smooth and fast graphic abilities are a must. Using large-size, superbquality images from a $\mathrm{C}$-arm implies that a powerful processing unit is needed.

Images from a $\mathrm{C}$-arm are stored in DICOM format files, which can then be used for biometric post processing, such as quantitative vessel analysis or 3D reconstruction. The higher the quality of the images obtained from the C-arm, the larger the size of the files that have to be processed by the workstation.

\section{Sterility Issues}

In order to guarantee highest flexibility in room usage, hospitals tend to equip all operating rooms according to the highest design standards which should include a laminar air flow ceiling. Some hospitals even require skirts around the laminar air flow field and this setup may preclude ceiling- mounted systems. In any case, ceiling-mounted systems with running parts above the operating field, which are difficult to clean and interfere with the air flow by causing turbulences, are least recommended from a hygienic stand point. ${ }^{12}$ It is very important to maintain sterility by having an effective HVAC system with well-developed pressure gradient especially in cases of intraoperative MRI (same machine can be utilized by radiology department).

\section{Hybrid OR with Intraoperative CT}

The system consists of a radiolucent adjustable, flexible, rotating operating table, an initially mobile and later ceiling-mounted frameless infrared-based neurosurgery navigation system (NNS), and a commercially available latest generation 40-multislice CT scanner with a sliding gantry and enlarged bore diameter of $82 \mathrm{~cm}$. The CT scanner is directly connected to the NNS, which allow automated referencing after data transfer. To increase patient safety, a sliding gantry concept is implemented that allows the operating table to remain in a stable position during imaging with the gantry moving over the radiolucent surgical table. The carbon- made OR table allows the patient to be placed in any position including prone, supine, park bench and sitting. Scanning is possible in all positions except sitting. The scanner is controlled by a workstation in a room next to the OR with direct visual contact and video surveillance. This setting enables $\mathrm{CT}$ image acquisition without any radiation exposure to personnel because personnel are not required to stay in the OR during image acquisition. ${ }^{17}$

All of this equipment is typically fixed in the suite and allows for ready utilization to produce very accurate highresolution image quality.

\section{Hybrid OR with Intraoperative MRI}

Intraoperative MRI was introduced in 1993. Since then, it has been generally accepted as a valuable image guidance

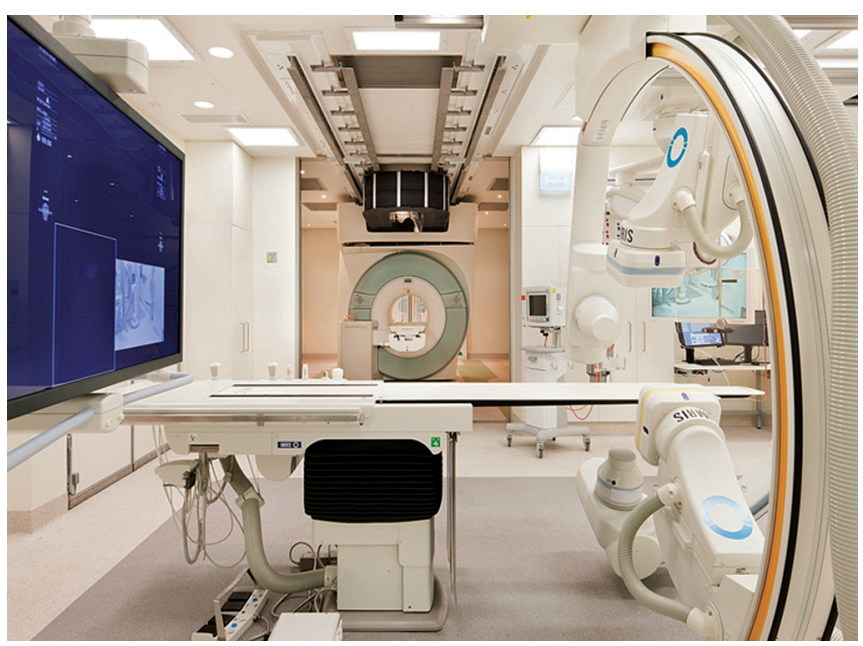

Fig. 2: Intraoperative magnetic resonance imaging 


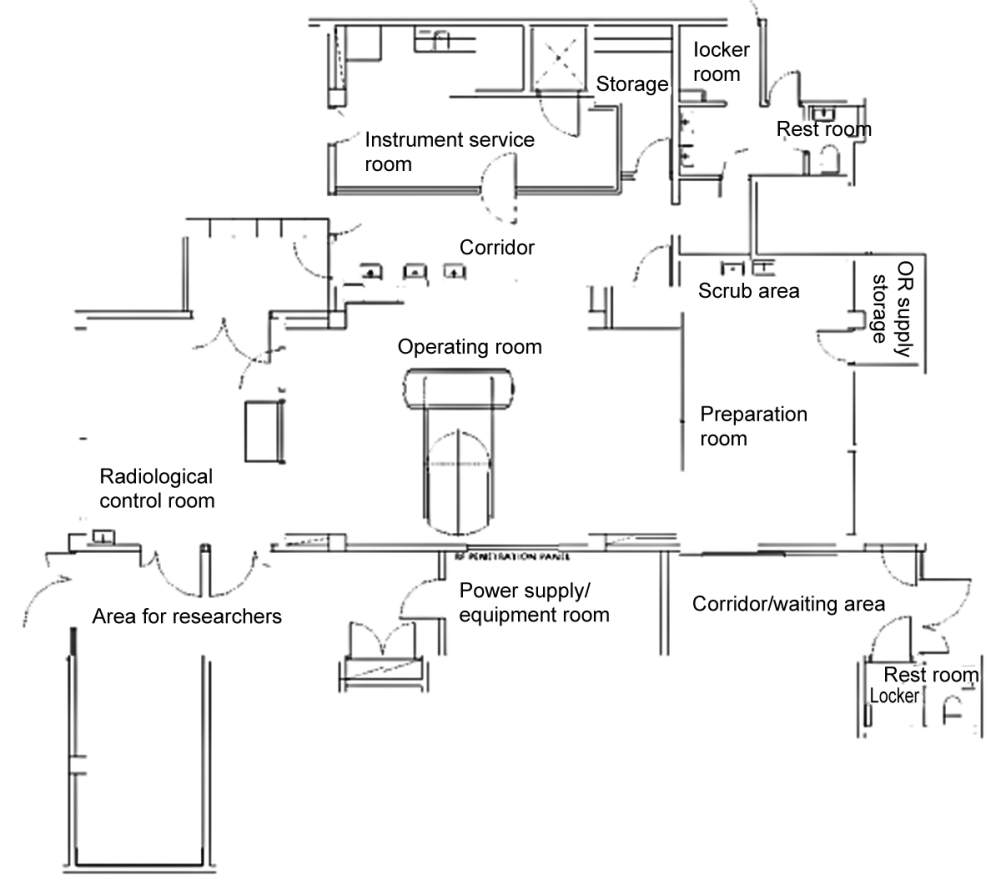

Fig. 3: Layout of the intraoperative MRI facilities at Oulu University Hospital ${ }^{18}$

tool for neurosurgery, but it still applies relatively immature and diverse technologies; its clinical indications are not well defined, and its potential impact on everyday neurosurgical practice is not yet fully recognized (Fig. 2).

Intraoperative magnetic resonance imaging (intraoperative MRI) is a promising method for image-guidance in minimally invasive neurosurgery. However, effective use of an intraoperative MRI unit requires new concepts that combine imaging and surgical environments. A compromise has to be sought between scanner design and imaging properties. The disadvantages of the method include the need for MRI-compatible instruments and devices, trained personnel to avoid accidents, a radiofrequency (RF)-shielded OR, and the necessary investment for the scanner. ${ }^{18}$

The reason for the early acceptance of intraoperative MRI is that it is not a so-called 'disruptive technology,' which necessitates the total transformation of a medical specialty. It has been easy to accept intraoperative guidance by MRI because it uses the same imaging modality for localization during surgery as it does for preoperative diagnosis. It also improves the now universally used intraoperative navigation by real-time, interactive, near-real-time imaging, with frequent volumetric updates. The main reason for the relatively slow proliferation of this technology is not necessarily the high cost of MRI systems but the lack of clear definition of the requirements of the various types of intraoperative MRI systems. ${ }^{19}$

Most of revised MRI facilities have been designed for use either as radiological intervention suites or as operating rooms (Fig. 3). The objective in planning the facilities was joint use by neurosurgeons and radiologists to allow frequent and varied use of the scanner. This made it necessary to create not only a unique operating environment but also an organizational model for administration of the IMRI Unit. Diagnostic imaging is supervised directly by the department of diagnostic radiology. For clinical intraoperative/ interventional work as well as research a coordinating board may be established. ${ }^{18}$

Brainsuite is an integrated neurosurgery system that allows for more precise treatment of complicated tumors in sensitive areas of the brain. Brainsuite is the latest advancement in image-guided surgery, providing real-time views of the tumor site with intraoperative magnetic resonance imaging (iMRI). ${ }^{20}$

\section{Future Perspectives $^{12}$}

1. Wireless devices will become reality in the near future and will overcome the direct limitations now present due to wire connection points.

2. Integration of robotic and navigational techniques into clinical practice may lead to improved catheter accuracy, stability, and safety in comparison with conventional techniques, while minimizing radiation exposure.

\section{CONCLUSION}

By maximizing the use of existing technologies while developing new approaches to treating these challenging cases, we hope that these would lead to improved overall clinical outcomes and further reduce the mortality and morbidity 
rates associated with managing the cardiovascular patient. It is hoped that as these new fields develop and with increasing experience with these new hybrid methods, we may well be able to maximize the applicability of minimally invasive endovascular and hybrid technology to treat a larger cohort of patients with cardiovascular disease.

\section{REFERENCES}

1. Towers M. The modern conception of an operating theatre suite. South African Med J 1967 Feb 25;41(8):181-186.

2. Gupta S, Kant S, Chandrashekhar R. Operating unit-planning essentials and design considerations. J Acad Hosp Adm 2005, Available at: http://www.indmedica.com/journals.php/12?jour nalid $=6 \&$ issueid $=72 \&$ articleid $=907 \&$ action $=$ article .

3. Van Pelt J. Hybrid ORs: what's behind the increasing demand? OR Manager 2011 May;27(5):7-10. Available at: http://www. ncbi.nlm.nih.gov/pubmed/21678825.

4. Stanbridge DD, Fried GM. Current developments in operating room design. Oper Room 2007;8-9. Available at: http://www. touchbriefings.com/pdf/2742/fried.pdf.

5. Portou MJ, Browne TF, Prionidis I. The vascular hybrid operating theatre: The Broomfield Hospital experience. RAD Mag 2011;29-31. Available at: http://www.radmagazine.co.uk/ ScientificPDFs/October 2011 - The vascular operating theatreDr M J Portou.pdf

6. Field ML, Sammut J, Kuduvalli M, Oo A, Rashid A. Hybrid theatres: nicety or necessity? JR Soc Med 2009 Mar;102(3): 92-97.

7. Fornell D. Planning for a hybrid suite 2010. Available at: http:// www.healthcare.philips.com

8. Belkin M. The design and implementation of hybrid operating rooms [cited 2011 Dec 29].p.3. Available at: www.veithsymposium.org/pdf/vei/2761.pdf
9. Kpodonu J, Raney A. The cardiovascular hybrid room a key component for hybrid interventions and image guided surgery in the emerging specialty of cardiovascular hybrid surgery. Interact Cardiovasc Thorac Surg 2009 Oct;9(4):688-692.

10. Naus H. Designing the hybrid OR considerations and recommendations. Philips Healthc p. 1-10.

11. Healthcare design insights. Surg Innov A/E/C Impacts. 2010.p.3. Available at: http://www.mahlum.com/pdf/MahlumHDISpring2010Issue02.pdf

12. Kpodonu J. Hybrid cardiovascular suite: the operating room of the future. J Card Surg 2010 Nov;25(6):704-709.

13. Mansour MA. The new operating room environment. Surg Clin North Am 1999 Jun;79(3):477-487.

14. Sikkink CJJM, Reijnen MMPJ, Zeebregts CJ. The creation of the optimal dedicated endovascular suite. Eur J Vasc Endovasc Surg 2008 Feb;35(2):198-204.

15. Fillinger MF, Weaver JB. Imaging equipment and techniques for optimal intraoperative imaging during endovascular interventions. Semin Vasc Surg 1999 Dec;12(4):315-326.

16. Cardiovascular imaging. Cardiovasc J Afr 2009 [cited 2012 Oct 17];20(4):258-259. Available at: http://www.cvja.co.za/onlinejournal/vol20/vol20_issue_4/index.html?pageNumber $=48$

17. Uhl E, Zausinger S, Morhard D, Heigl T, Scheder B, Rachinger $\mathrm{W}$, et al. Intraoperative computed tomography with integrated navigation system in a multidisciplinary operating suite. Neurosurgery 2009 May;64(5 Suppl 2):231-239.

18. Yrjänä SK, Katisko JP, Ojala RO, Tervonen O, Schiffbauer H, Koivukangas J Versatile intraoperative MRI in neurosurgery and radiology. Acta Neurochir 2002 Mar;144(3):271-278.

19. Jolesz Fa. Future perspectives for intraoperative MRI. Neurosurg Clin N Am 2005 Jan;16(1):201-213.

20. Brain \& Spine Center - Brainsuite / MD Anderson Cancer Center. Available at: http://www.mdanderson.org/patient-andcancer-information/care-centers-and-clinics/care-centers/ brain-spine/services/index.html. 